\title{
Statement of the American College of Medical Genetics on Universal Newborn Hearing Screening
}

Hearing loss is present at birth in 1-2 per 1,000 infants. Physiologic newborn hearing screening tests are now available that have high sensitivity when properly administered. Additionally, studies have shown that early intervention to facilitate the development of oral or manual communication can have a profound influence on the successful acquisition of language by deaf children. These developments have led to a growing recognition of the value of universal newborn hearing screening that has been articulated in the Year 2000 Position Statement of the Joint Committee on Infant Hearing and endorsed by relevant professional organizations including the American Speech, Language \& Hearing Association, the American Academy of Pediatrics, and with this Statement, the American College of Medical Genetics.

In addition to these important developments, dramatic recent advances have been made in our understanding of the specific causes of clinically significant hearing loss. Deafness is an etiologically heterogeneous trait with many known genetic and environmental causes that can often be identified by a family history, clinical findings, and specific DNA-based diagnostic tests. At least $50 \%$ of all profound hearing loss can be attributed to the effects of single genes or gene pairs at many different loci. More than 50 genes for human hearing loss have been mapped and a growing number of these have been cloned. Reliable DNA-based screening tests have been developed to detect common genetic forms of deafness such as Connexin 26 and mitochondrial deafness.

In addition to nonsyndromic forms of deafness in which hearing loss is the only clinical finding, a large number of syndromic forms are recognized in which there is associated involvement of other tissues or organs. Establishing an accurate genetic diagnosis can therefore be of great benefit to the patient and family in establishing an etiology and the prevention of adverse clinical sequelae. Because of the genetic complexity of this phenotype and the lack of complete knowledge about the prognostic significance of the genetic test results that are becoming available, we feel it is essential that all children with confirmed hearing loss be referred for evaluation and genetic counseling to a team that will typically include a qualified clin-

\begin{abstract}
ical geneticist and a genetic counselor. Geneticists are uniquely qualified by their training and profession to understand and deal with the nuances of these genetic tests and to convey genetic information and the significance of test results to their patients in a responsible manner. During the next decade, new tests to detect other forms of genetic deafness are certain to appear and if the benefits of these tests are to be realized, they should be made available to the public through professionals who fully understand their implications and limitations. Although the details of individual screening programs may differ, they must all address the general issues that have been identified in published guidelines for existing Newborn Screening Programs. These include (1) careful attention to maximizing the sensitivity and specificity not only of the primary screening test(s) but subsequent diagnostic tests to confirm the presence of a hearing loss and then to establish the etiology; (2) the development of protocols that ensure successful and timely follow up of all patients who do not pass the initial screening test; (3) the provision of appropriate clinical, audiologic, and genetic follow up, evaluation, and testing not only to confirm the presence of a hearing loss but to establish the etiology whenever possible; (4) coordinated planning for case management and therapeutic intervention that should include counseling about the significance of test results and the available therapeutic options; and (5) ongoing evaluation of the validity, efficiency, and overall benefit of the program to the patient, the family, and society. Public health hearing-screening databases should be linked to other related public health databases. All states now screen all newborns for selected metabolic disorders. Over the years, these programs have solved many of the difficult logistic problems involved in education, record keeping, follow up tracking, referrals, and clinical management of the high risk infants detected through these programs. To the maximum extent possible, hearing screening programs should exploit the experience and knowledge these programs have acquired.
\end{abstract}

Reviewed and approved by the ACMG Board of Directors, January 2000.

This guideline is designed primarily as an educational resource for medical geneticists and other health care providers to help them provide quality medical genetic services. Adherence to this guideline does not necessarily assure a successful medical outcome. This guideline should not be considered inclusive of all proper procedures and tests or exclusive of other procedures and tests that are reasonably directed to obtaining the same results. In determining the propriety of any specific procedure or test, the geneticists should apply his or her own professional judgment to the specific clinical circumstances presented by the individual patient or specimen. It may be prudent, however, to document in the patient's record the rationale for any significant deviation from this guideline. 


\section{policystatement}

This statement was prepared by the Newborn Hearing Subcommittee of the Public Health Genetics Committee (PHGC) and was approved by the Board of Directors of the American College of Medical Genetics in January 2000.

Walter E. Nance, $M D, P h D$ (Chairman)

George C. Cunningham, MD, MPH (Chairman, PHGC)

Jessica G. Davis, MD

Cynthia C. Morton, PhD

Louis J. Elsas, MD

Teresa Finitzo, PhD

Rena E. Falk, $M D$
Paul S. Ing, PhD

Arti Pandya, $M D, M B A$

Edward R.B. McCabe, MD, PhD

Richard J.H. Smith, MD

(C) American College of Medical Genetics, 2000

AMERICAN COLLEGE OF MEDICAL GENETICS

9650 Rockville Pike

Bethesda, Maryland 20814-3998

Phone: 301-530-7127

Fax: 301-571-1895 